PROCEEDINGS OF THE

AMERICAN MATHEMATICAL SOCIETY

Volume 138, Number 3, March 2010, Pages 943-951

S 0002-9939(09)10106-5

Article electronically published on October 23, 2009

\title{
HYPERGEOMETRIC ORIGINS OF DIOPHANTINE PROPERTIES ASSOCIATED WITH THE ASKEY SCHEME
}

\author{
YANG CHEN AND MOURAD E. H. ISMAIL
}

(Communicated by Walter Van Assche)

\begin{abstract}
The "Diophantine" properties of the zeros of certain polynomials in the Askey scheme, recently discovered by Calogero and his collaborators, are explained, with suitably chosen parameter values, in terms of the summation theorem of hypergeometric series. Here the Diophantine property refers to integer valued zeros. It turns out that the same procedure can also be applied to polynomials arising from the basic hypergeometric series. We found, with suitably chosen parameters and certain $q$-analogues of the summation theorems, zeros of these polynomials explicitly which are no longer integer valued. This goes beyond the results obtained by the authors previously mentioned.
\end{abstract}

\section{INTRODUCTION}

In a series of papers Calogero and his collaborators (see for example [3, 4, and [5]) investigated various integrable lattices of the Toda-type with suitable boundary conditions. These lattices arose as the dressing chains of Adler, Shabat, Yamilov and others. See for example [1, [13] and [16. It is found that if the small amplitude motion about the equilibrium configuration is assumed to be isochronous, namely, each component is periodic with the same period, then the characteristic frequencies must necessarily have integer values. Furthermore, if the assumption of nearest neighbor interaction is made in the lattice models, then the secular equation whose zeros give the characteristic frequencies reads

$$
\operatorname{det}\left(x \mathrm{I}_{N}-\mathrm{A}_{N}\right)=0
$$

where $\mathrm{A}_{N}$ is a tri-diagonal matrix of size $N$. We may take $N$ to be the number of particles in the many-body problem. See [7] for a detailed treatment. Hence

$$
P_{N}(x):=\operatorname{det}\left(\left.x\right|_{N}-\mathrm{A}_{N}\right)
$$

may be interpreted as orthogonal polynomials if the super-diagonal elements of $\mathrm{A}_{N}$ are real and none of them vanishes. We show that the Diophantine properties arise when the parameters of the orthogonal polynomials are suitably chosen. The factorization occurs when the polynomials, although still characteristic polynomials of tri-diagonal matrices, are no longer orthogonal.

Received by the editors March 7, 2009, and, in revised form, June 12, 2009.

2000 Mathematics Subject Classification. Primary 33C20, 33C45.

Key words and phrases. Generalized hypergeometric series, basic hypergeometric series, summation theorems.

(C)2009 American Mathematical Society Reverts to public domain 28 years from publication 
The motivation of considering this problem came from reading [6], where a hypergeometric polynomial of degree $n$ is factored as $f_{m}(x) g_{n-m}(x)$. Here $f_{m}$ has degree $m, g_{n-m}$ has degree $n-m$ and the zeros of $f_{m}$ are squares of equi-spaced points. This holds for all $m, 1 \leq m \leq n$. This factorization is referred to as having the "Diophantine property" in 6]. Our explanation is that all the Diophantine results in 6] follow from summation theorems for hypergeometric functions. This will be shown in $\S 3$. In $\S 4$ we provide $q$-analogues of all the results of $\S 3$, that is, all the results in 6. Section 2 contains the notation, summation theorems, and transformation formulas used in $\S 3$ and $\S 4$.

The zeros of the polynomial $f_{m}$ in the factorization of the Askey-Wilson polynomials given in $\S 4$ are the points $\left[a q^{k}+q^{-k} / a\right] / 2, k=0,1,2, \cdots$, where $a$ is one of the parameters in the Askey-Wilson polynomial. Such points after $a \rightarrow i a$ are interpolation points in the sense that the values of an entire function $h$ at these points determine the function uniquely provided that $M(h, r)$, the maximum modulus of $h$, satisfies $M(h, r) \leq C r^{\alpha} \exp \left(b(\ln r)^{2}\right)$ with $-b \ln q<1$, for some $\alpha$; see [11]. The integers are interpolation points for entire functions $h$ for which $M(h, r) \leq C \exp (b r)$ with $b<\pi$.

It is known that a sequence of monic orthogonal polynomials satisfies a three term recurrence relation

$$
x P_{n}(x)=P_{n+1}(x)+\alpha_{n} P_{n}(x)+\beta_{n} P_{n-1}(x), n>0,
$$

with $P_{0}(x):=1, P_{1}(x):=x-\alpha_{0}$. The monic polynomials have the determinant representation

$$
P_{n}(x)=\left|\begin{array}{ccccccc}
x-\alpha_{0} & -a_{1} & 0 & \cdots & 0 & 0 & 0 \\
-a_{1} & x-\alpha_{1} & -a_{2} & \cdots & 0 & 0 & 0 \\
\vdots & \ddots & \ddots & \ddots & \vdots & \vdots & \vdots \\
0 & 0 & \ldots & & -a_{n-2} & x-\alpha_{n-2} & -a_{n-1} \\
0 & 0 & 0 & \cdots & 0 & -a_{n-1} & x-\alpha_{n-1}
\end{array}\right|,
$$

where $a_{n}^{2}=\beta_{n}, n>0$. It is clear that if $\beta_{k}=0$ for some $k<n$, then $P_{n}(x)$ factors into a product of two polynomials of the same type, that is, a product of two characteristic polynomials of tri-diagonal matrices. All the factorizations in the work of Bruschi, Calogero, and Droghei are of this type. What is surprising is that one of the two characteristic polynomials has equi-spaced zeros. In $\S 5$ we give another conceptual explanation of the results in this work which will explain the origin of the results of Calogero and his collaborators.

Remark 1.1. It is important to note that the Wilson polynomials are believed to be the most general orthogonal polynomials of hypergeometric type, while the Askey-Wilson polynomials are the most general orthogonal polynomials of basic hypergeometric type. As such we believe that it is unlikely this work can be extended to more general polynomials.

\section{Summation theorems}

Recall that the $q$-shifted factorial is

$$
(a ; q)_{n}=\prod_{k=1}^{n}\left(1-a q^{k-1}\right)
$$


and a basic hypergeometric function is

$$
{ }_{r+1} \phi_{r}\left(\begin{array}{c}
a_{1}, a_{2}, \cdots, a_{r+1} \\
b_{1}, b_{2}, \cdots, b_{r}
\end{array} \mid q, z\right)=\sum_{n=0}^{\infty} \prod_{k=1}^{r+1} \frac{\left(a_{k} ; q\right)_{n}}{\left(b_{k-1} ; q\right)_{n}} z^{n},
$$

where $b_{0}:=q$.

The Pfaff-Saalschütz theorem is

$$
{ }_{3} F_{2}\left(\begin{array}{c}
-n, \quad A, \quad B \\
C, 1+A+B-n-C
\end{array} \mid 1\right)=\frac{(C-A)_{n}(C-B)_{n}}{(C)_{n}(C-A-B)_{n}} .
$$

The summation formula

$$
\begin{gathered}
{ }_{r+2} F_{r+1}\left(\begin{array}{c}
A, B, B_{1}+m_{1}, \cdots, B_{r}+m_{r} \\
B+1, B_{1}, \cdots, B_{r}
\end{array} \mid 1\right) \\
=\frac{\Gamma(B+1) \Gamma(1-A)}{\Gamma(1+B-A)} \prod_{j=1}^{r} \frac{\left(B_{j}-B\right)_{m_{j}}}{\left(B_{j}\right)_{m_{j}}}
\end{gathered}
$$

is known as the Karlsson-Minton sum [9, but it follows from the earlier work of Fields and Wimp [8]. In particular we have

$$
{ }_{r+1} F_{r}\left(\begin{array}{c|c}
A, B_{1}+m_{1}, \cdots, B_{r}+m_{r} & 1 \\
B_{1}, \cdots, B_{r} & 1
\end{array}\right)=0
$$

for $\operatorname{Re}(-A)>m_{1}+m_{2}+\cdots+m_{r}$. We will also apply the Whipple transformation 14,

$$
\begin{aligned}
& { }_{4} F_{3}\left(\begin{array}{c}
-n, A, B, C \\
D, E, F
\end{array} \mid 1\right)=\frac{(E-A)_{n}(F-A)_{n}}{(E)_{n}(F)_{n}} \\
& \times{ }_{4} F_{3}\left(\begin{array}{c}
-n, A, D-B, D-C \\
D, A+1-n-E, A+1-n-F
\end{array} \mid 1\right),
\end{aligned}
$$

where $D+E+F=A+B+C+1-n$.

The $q$-analogue of the Pfaff-Saalschütz theorem is

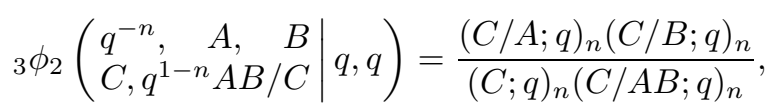

while the $q$-analogue of the Whipple transformation is the Sears transformation [9, (III.15)],

$$
\begin{array}{r}
{ }_{4} \phi_{3}\left(\begin{array}{c}
q^{-n}, A, B, C \\
D, E, F
\end{array} \mid q, q\right)=A^{n} \frac{(E / A ; q)_{n}(F / A ; q)_{n}}{(E ; q)_{n}(F ; q)_{n}} \\
\times{ }_{4} \phi_{3}\left(\begin{array}{c}
q^{-n}, A, D / B, D / C \\
D, q^{1-n} A / E, q^{1-n} A / F
\end{array} \mid q, q\right),
\end{array}
$$

where $D E F=q^{1-n} A B C$.

Some useful identities are

$$
\begin{gathered}
\left(a q^{-n} ; q\right)_{n}=(q / a ; q)_{n}(-a)^{n} q^{-\left(\begin{array}{c}
n+1 \\
2
\end{array}\right)}, \\
\left(a q^{-n} ; q\right)_{n-k}=\frac{(q / a ; q)_{n}}{(q / a ; q)_{k}}(-a)^{n-k} q^{\left(\begin{array}{c}
k+1 \\
2
\end{array}\right)-\left(\begin{array}{c}
n+1 \\
2
\end{array}\right) .}
\end{gathered}
$$

Of course the first is a special case of the second. 


\section{Complete factorization of the Wilson AND RELATED POLYNOMIALS}

The Wilson polynomials are

$$
\begin{gathered}
W_{n}(x ; \mathbf{t})=\prod_{j=1}^{3}\left(t_{1}+t_{j}\right)_{n} \\
\times_{4} F_{3}\left(\begin{array}{c}
-n, t_{1}+t_{2}+t_{3}+t_{4}+n-1, t_{1}+i \sqrt{x}, t_{1}-i \sqrt{x} \\
t_{1}+t_{2}, t_{1}+t_{3}, t_{1}+t_{4}
\end{array} \mid 1\right),
\end{gathered}
$$

where $\mathbf{t}:=\left(t_{1}, t_{2}, t_{3}, t_{4}\right)$. It is a fact that the Wilson polynomials are symmetric in the parameters $t_{1}, t_{2}, t_{3}, t_{4}$. The invariance of $W_{n}$ under permutations of $\left\{t_{2}, t_{3}, t_{4}\right\}$ is obvious, but the invariance under permuting $t_{1}$ and $t_{j}$ for $j=2,3,4$ is not obvious and is called the Whipple transformation [14.

If we wish to find a complete factorization of $W_{n}$ or equivalently identify all the zeros of $W_{n}$, then we must choose the parameters in such a way that the ${ }_{4} F_{3}$ representation can be summed explicitly.

We shall denote the monic Wilson polynomials by $\left\{\tilde{W}_{n}(x ; \mathbf{t})\right\}$; that is,

$$
\begin{gathered}
\tilde{W}_{n}\left(x ; t_{1}, t_{2}, t_{3}, t_{4}\right) \\
=\frac{(-1)^{n}}{\left(n+t_{1}+t_{2}+t_{3}+t_{4}-1\right)_{n}} W_{n}\left(x ; t_{1}, t_{2}, t_{3}, t_{4}\right) .
\end{gathered}
$$

Case 1 . We reduce the ${ }_{4} F_{3}$ to ${ }_{3} F_{2}$ and use the Pfaff-Saalschütz theorem. Since we want to keep $x$ in the factorization, we demand that $n-1+\sum_{k=1}^{4} t_{k}$ be equal to $t_{1}+t_{j}$ for some $j$. It is easy to see that this happens if and only if $t_{i}+t_{j}=1-n$ for some $i \neq j, 1<i, j \leq 4$. There is no loss of generality in assuming $t_{4}=1-n-t_{3}$. In this case (2.3) gives

$$
\begin{gathered}
\tilde{W}_{n}\left(x ; t_{1}, t_{2}, t_{3}, 1-n-t_{3}\right)=(-1)^{n}\left(t_{1}+t_{3}\right)_{n}\left(t_{1}+1-n-t_{3}\right)_{n} \\
\quad \times_{3} F_{2}\left(\begin{array}{c}
-n, t_{1}+i \sqrt{x}, t_{1}-i \sqrt{x} \\
t_{1}+t_{3}, t_{1}+1-n-t_{3}
\end{array} \mid\right) \\
=(-1)^{n}\left(t_{1}+t_{3}\right)_{n}\left(t_{1}+1-n-t_{3}\right)_{n} \times \frac{\left(t_{3}+i \sqrt{x}\right)_{n}\left(t_{3}-i \sqrt{x}\right)_{n}}{\left(t_{1}+t_{3}\right)_{n}\left(t_{3}-t_{1}\right)_{n}} .
\end{gathered}
$$

Since $(-1)^{n}\left(t_{1}-t_{3}+1-n\right)_{n}=\left(t_{3}-t_{1}\right)_{n}$, it follows that

$$
\begin{gathered}
\tilde{W}_{n}\left(x ; t_{1}, t_{2}, t_{3}, 1-n-t_{3}\right)=\left(t_{3}+i \sqrt{x}\right)_{n}\left(t_{3}-i \sqrt{x}\right)_{n} \\
=\prod_{k=1}^{n}\left[x^{2}+\left(t_{3}+k-1\right)^{2}\right] .
\end{gathered}
$$

The above factorization is equations (33) and (41a) of [6].

Case 2. We identify values of $x$ suitable to apply (2.5). For example, we may choose $i \sqrt{x}=t_{4}+j$ and make $t_{1}-i \sqrt{x}$, which is $t_{1}-t_{4}-j$, equal $t_{1}+t_{3}+k$. Thus we make the parameter identification $x=-\left(t_{4}+j\right)^{2}, t_{3}=-t_{4}-j-k$. Finally we demand that $n+t_{3}+t_{4}-1=s$, which is equivalent to $n-1 \geq j+k$. Now we set $m=j+k+1$, replace $j$ by $j-1$ and find that

$$
W_{n}\left(-\left(t_{4}+j-1\right)^{2} ; t_{1}, t_{2},-t_{4}-m+1, t_{4}\right)=0,
$$


for $1 \leq j \leq m, 1 \leq m \leq n$. This is (35) in [6]. This is particularly interesting because it seems to give a partial factorization when $m<n$. We shall return to this point at the end of this section.

When $m=n$ we obtain the factorization

$$
\tilde{W}_{n}\left(x ; t_{1}, t_{2},-t_{4}-n+1, t_{4}\right)=\prod_{j=1}^{n}\left[x+\left(t_{4}+j-1\right)^{2}\right],
$$

which is $(34)$ in [6]. The special case $t_{4}=(1-2 n) / 4$ is $(41 \mathrm{a})$ of [6]. This last factorization also follows from the Pfaff-Saalschütz formula since the ${ }_{4} F_{3}$ reduces to ${ }_{3} F_{2}$. Indeed in this case we have

$$
\begin{aligned}
W_{n}\left(x ; t_{1}, t_{2},-t_{4}\right. & \left.-n+1, t_{4}\right)=\left(t_{1}+t_{2}\right)_{n}\left(t_{1}+t_{4}\right)_{n}\left(t_{1}+1-n-t_{4}\right)_{n} \\
& \times{ }_{3} F_{2}\left(\begin{array}{c}
-n, t_{1}+i \sqrt{x}, t_{1}-i \sqrt{x} \\
t_{1}+t_{4}, t_{1}+1-n-t_{4}
\end{array} \mid 1\right) .
\end{aligned}
$$

The Pfaff-Saalschütz theorem implies

$$
\begin{aligned}
& W_{n}\left(x ; t_{1}, t_{2},-t_{4}-n+1, t_{4}\right) \\
& \quad=(-1)^{n}\left(t_{1}+t_{4}\right)_{n}\left(t_{4}+i \sqrt{x}\right)_{n}\left(t_{4}-i \sqrt{x}\right)_{n},
\end{aligned}
$$

after some manipulations.

We now show how to discover (3.4) from the Whipple transformation. It is clear that $W_{n}\left(x ; t_{1}, t_{2}, 1-t_{4}-m, t_{4}\right)$ is a constant multiple of

$$
\begin{gathered}
{ }_{4} F_{3}\left(\begin{array}{c|c}
-n, t_{1}+t_{2}+n-m, t_{1}+i \sqrt{x}, t_{1}-i \sqrt{x} \\
t_{1}+t_{2}, t_{1}+1-m-t_{4}, t_{1}+t_{4}
\end{array} \mid 1\right) \\
=\frac{\left(1-m-t_{4}-i \sqrt{x}\right)_{n}\left(t_{4}-i \sqrt{x}\right)_{n}}{\left(t_{1}-t_{4}+1-m\right)_{n}\left(t_{1}+t_{4}\right)_{n}} \\
\times{ }_{4} F_{3}\left(\begin{array}{c}
-n, \quad t_{1}+i \sqrt{x}, m-n, \quad t_{2}+i \sqrt{x} \\
t_{1}+t_{2}, t_{4}+i \sqrt{x}+m-n, i \sqrt{x}+1-n-t_{4}
\end{array}\right) .
\end{gathered}
$$

In the last step we applied the Whipple transformation (2.6) with the parameter identification

$$
\begin{gathered}
A=t_{1}+i \sqrt{x}, B=n+t_{1}+t_{2}-m, C=t_{1}-i \sqrt{x}, \\
D=t_{1}+t_{2}, E=t_{1}-t_{4}+1-m, F=t_{1}+t_{4} .
\end{gathered}
$$

We next apply the Whipple transformation again with the choices

$$
\begin{gathered}
A=t_{2}+i \sqrt{x}, B=t_{1}+i \sqrt{x}, C=-n, \\
D=t_{1}+t_{2}, E=t_{4}+i \sqrt{x}+m-n, F=1-n-t_{4}+i \sqrt{x},
\end{gathered}
$$

and $n$ is now $n-m$. Therefore the left-hand side of (3.7) is

$$
\begin{gathered}
\frac{\left(1-m-t_{4}-i \sqrt{x}\right)_{n}\left(t_{4}-i \sqrt{x}\right)_{n}}{\left(t_{1}-t_{4}+1-m\right)_{n}\left(t_{1}+t_{4}\right)_{n}} \\
\times \frac{\left(t_{4}-t_{2}+m-n\right)_{n-m}\left(1-n-t_{2}-t_{4}\right)_{n-m}}{\left(t_{4}+m-n+i \sqrt{x}\right)_{n-m}\left(1-n-t_{4}+i \sqrt{x}\right)_{n-m}} \\
\times{ }_{4} F_{3}\left(\begin{array}{cc|c}
m-n, \quad n+t_{1}+t_{2}, \quad t_{2}+i \sqrt{x}, \quad t_{2}-i \sqrt{x} & 1 \\
t_{1}+t_{2}, t_{2}+1-t_{4}, t_{2}+t_{4}+m & 1
\end{array}\right) .
\end{gathered}
$$


It is clear that the ${ }_{4} F_{3}$ in the above expression is a constant multiple of $W_{n-m}\left(x ; t_{2}, t_{1}, 1-t_{4}, m+t_{4}\right)$. We now apply the identities

$$
(\alpha+1)_{n}=(-1)^{n}(-\alpha-n)_{n}, \quad(\alpha)_{n}=(\alpha)_{m}(\alpha+m)_{n-m}
$$

to see that

$$
\begin{gathered}
\frac{\left(1-m-t_{4}-i \sqrt{x}\right)_{n}\left(t_{4}-i \sqrt{x}\right)_{n}}{\left(t_{4}+m-n+i \sqrt{x}\right)_{n-m}\left(1-n-t_{4}+i \sqrt{x}\right)_{n-m}} \\
=\frac{\left(1-m-t_{4}-i \sqrt{x}\right)_{n}\left(t_{4}-i \sqrt{x}\right)_{n}}{\left(1-t_{4}-i \sqrt{x}\right)_{n-m}\left(m+t_{4}-\sqrt{x}\right)_{n-m}} \\
=\frac{\left(1-m-t_{4}-i \sqrt{x}\right)_{m}\left(t_{4}-i \sqrt{x}\right)_{n}}{\left(m+t_{4}-\sqrt{x}\right)_{n-m}} \\
=(-1)^{m}\left(t_{4}+i \sqrt{x}\right)_{m}\left(t_{4}-i \sqrt{x}\right)_{m}=(-1)^{m} \prod_{j=0}^{m-1}\left[x^{2}+\left(t_{4}+j\right)^{2}\right] .
\end{gathered}
$$

This shows that

$$
\begin{gathered}
\frac{W_{n}\left(x ; t_{1}, t_{2}, 1-t_{4}-m, t_{4}\right)}{\left(t_{1}+t_{2}\right)_{n}\left(t_{1}+t_{2}\right)_{n}\left(1-t_{4}-m\right)_{n}\left(t_{1}+t_{4}\right)_{n}} \\
=\frac{(-1)^{m}\left(t_{4}+i \sqrt{x}\right)_{m}\left(t_{4}-i \sqrt{x}\right)_{m}}{\left(t_{1}+t_{2}\right)_{n-m}\left(t_{1}+t_{4}\right)_{n-m}\left(t_{1}-t_{4}+1-m\right)_{n-m}} \\
\times W_{n-m}\left(x ; t_{2}, t_{1}, 1-t_{4}, t_{4}+m\right) .
\end{gathered}
$$

\section{The Askey-Wilson polynomials}

The Askey-Wilson polynomials are defined through the representation

$$
\begin{aligned}
& p_{n}(x ; \mathbf{t})=t_{1}^{-n} \prod_{j=1}^{3}\left(t_{1} t_{j} ; q\right)_{n} \\
& \times_{4} \phi_{3}\left(\begin{array}{c|c}
q^{-n}, t_{1} t_{2} t_{3} t_{4} q^{n-1}, t_{1} e^{i \theta}, t_{1} e^{-i \theta} \\
t_{1} t_{2}, t_{1} t_{3}, t_{1} t_{4}
\end{array} \mid q, q\right) .
\end{aligned}
$$

As in Case 1 of $\S 3$ we reduce the ${ }_{4} \phi_{3}$ to ${ }_{3} \phi_{2}$. There is no loss of generality in assuming that $q^{n-1} t_{1} t_{2} t_{3} t_{4}=t_{1} t_{2}$, that is, $t_{4}=q^{n-1} / t_{3}$. Applying the summation formula (2.7) we get

$$
\begin{gathered}
p_{n}\left(\cos \theta ; t_{1}, t_{2}, t_{3}, q^{n-1} / t_{3}\right) \\
=\left(t_{3} e^{i \theta} ; q\right)_{n}\left(t_{3} e^{-i \theta} ; q\right)_{n} \frac{\left(t_{1} t_{2} ; q\right)_{n}\left(q^{1-n} t_{1} / t_{3} ; q\right)_{n}}{t_{1}^{n}\left(t_{3} / t_{1} ; q\right)_{n}} \\
=\left(-t_{3}\right)^{-n} q^{-\left(\begin{array}{c}
n \\
2
\end{array}\right)\left(t_{3} e^{i \theta} ; q\right)_{n}\left(t_{3} e^{-i \theta} ; q\right)_{n}\left(t_{1} t_{2} ; q\right)_{n} .}
\end{gathered}
$$

It is clear that

$$
\left(t_{3} e^{i \theta} ; q\right)_{n}\left(t_{3} e^{-i \theta} ; q\right)_{n}=\prod_{k=1}^{n}\left[1-2 t_{3} x q^{k-1}+t_{3}^{2} q^{2 k-2}\right],
$$

from which we can find the zeros explicitly.

As in Case 2 of $\S 3$ we choose $t_{3}=q^{1-m} / t_{4}$, then apply the Sears transformation (2.8) with the parameter identification

$$
\begin{gathered}
A=t_{1} e^{i \theta}, \quad B=q^{n-m} t_{1} t_{2}, \quad C=t_{1} e^{-i \theta}, \\
D=t_{1} t_{2}, \quad E=q^{1-m} t_{1} / t_{4}, \quad F=t_{1} t_{4} .
\end{gathered}
$$


The result is

$$
\begin{gathered}
p_{n}(\cos \theta ; \mathbf{t})=e^{i n \theta}\left(t_{1} t_{2} ; q\right)_{n}\left(q^{1-m} e^{i \theta} / t_{4} ; q\right)_{n}\left(t_{4} e^{-i \theta} ; q\right)_{n} \\
\quad \times_{4} \phi_{3}\left(\begin{array}{c}
q^{-n}, t_{1} e^{i \theta}, q^{m-n}, t_{2} e^{i \theta} \\
t_{1} t_{2}, q^{m-n} t_{4} e^{i \theta}, q^{1-n} e^{i \theta} / t_{4}
\end{array} \mid q, q\right) .
\end{gathered}
$$

We next apply the Sears transformation again with the choices

$$
\begin{gathered}
A=t_{2} e^{i \theta}, \quad B=t_{1} e^{i \theta}, \quad C=q^{-n}, \\
D=t_{1} t_{2}, \quad E=t_{4} q^{m-n} e^{i \theta}, \quad F=q^{1-n} e^{i \theta} / t_{4},
\end{gathered}
$$

and the terminating parameter $n$ is replaced by $n-m$. This leads to

$$
\begin{gathered}
p_{n}(x ; \mathbf{t})=\frac{t_{2}^{2(n-m)}\left(t_{1} t_{2} ; q\right)_{n}\left(q^{1-m} e^{-i \theta} / t_{4} ; q\right)_{n}\left(t_{4} e^{-i \theta} ; q\right)_{n}}{\left(t_{1} t_{2} ; q\right)_{n-m}\left(t_{2} q / t_{4} ; q\right)_{n-m}\left(q^{m} t_{2} t_{4} ; q\right)_{n-m}} \\
\times \frac{\left(q^{m-n} t_{4} / t_{2} ; q\right)_{n-m}\left(q^{1-n} / t_{2} t_{4} ; q\right)_{n-m}}{\left(q^{m-n} t_{4} q^{i \theta} ; q\right)_{n-m}\left(q^{1-n} e^{i \theta} / t_{4} ; q\right)_{n-m}} e^{i(2 n-m) \theta} \\
\times p_{n-m}\left(x ; t_{2}, t_{1}, q / t_{4}, q^{m} t_{4}\right),
\end{gathered}
$$

with $x=\cos \theta$. Applying equations (2.9) -(2.10) we finally establish the factorization

$$
\begin{gathered}
p_{n}\left(x ; t_{1}, t_{2}, q^{1-m} / t_{4}, t_{4}\right) \\
=\left(t_{4} e^{i \theta} ; q\right)_{m}\left(t_{4} e^{-i \theta} ; q\right)_{m} p_{n-m}\left(x ; t_{2}, t_{1}, q / t_{4}, q^{m} t_{4}\right) \\
\times(-1)^{m} t_{4}^{n-2 m} t_{2}^{m-n} \frac{\left(q^{m} t_{1} t_{2} ; q\right)_{n-m}}{\left(q^{m} t_{1} t_{4} ; q\right)_{n-m}},
\end{gathered}
$$

again with $x=\cos \theta$. Note that

$$
\left(t_{4} e^{i \theta} ; q\right)_{m}\left(t_{4} e^{-i \theta} ; q\right)_{m}=\prod_{k=1}^{n}\left[1-2 x t_{4} q^{k-1}+t_{4}^{2} q^{2 k-2}\right] .
$$

\section{REMARKS}

One referee pointed out a possible explanation of the results in this paper. We will illustrate this approach in the case of the Wilson polynomials. Let $\mu(x, \mathbf{t})$ be the measure of orthogonality of the Wilson polynomials $\left\{W_{n}(x ; \mathbf{t})\right\}$. The weight function is

$$
\mu^{\prime}(x, \mathbf{t})=w(x, \mathbf{t}):=\frac{\prod_{j=1}^{4} \Gamma\left(t_{j}+i \sqrt{x}\right) \Gamma\left(t_{j}-i \sqrt{x}\right)}{\Gamma(2 i \sqrt{x}) \Gamma(-2 i \sqrt{x})}, \quad x>0 .
$$

If all the $t$ parameters are positive, then the Wilson polynomials are orthogonal with respect to an absolutely continuous measure. When a parameter, say $t_{3}$, is negative, then the measure of orthogonality has a discrete part. Now consider the case $t_{j}>0, j=1,2, t_{3}=1-m-t_{4}, 0<t_{4}<1$. The discrete masses are at $x_{j}=-\left(t_{3}+j\right)^{2}, 0 \leq j<m$; hence $\left(t_{4}+i \sqrt{x}\right)_{m}\left(t_{4}-i \sqrt{x}\right)_{m}$ vanishes on the discrete part. On one hand, the orthogonal polynomials must be constant multiples of $\left\{W_{n}\left(x ; t_{1}, t_{2}, 1-m-t_{4}, t_{4}\right)\right\}$. On the other hand, using $\left(1-t_{4}-m \pm i \sqrt{x}\right)_{m}=$ $(-1)^{m}\left(t_{4} \mp i \sqrt{x}\right)_{m}$ we see that

$$
\begin{gathered}
\left(t_{4}+i \sqrt{x}\right)_{m}\left(t_{4}-i \sqrt{x}\right)_{m} \Gamma\left(t_{3}+i \sqrt{x}\right) \Gamma\left(t_{3}-i \sqrt{x}\right) \\
=\Gamma\left(1-t_{4}+i \sqrt{x}\right) \Gamma\left(1-t_{4}-i \sqrt{x}\right) .
\end{gathered}
$$


Therefore for polynomials $f$ we have

$$
\begin{gathered}
\int_{\mathbb{R}}\left[\left(t_{4}+i \sqrt{x}\right)_{m}\left(t_{4}-i \sqrt{x}\right)_{m}\right]^{2} f(x) d \mu\left(x, t_{1}, t_{2}, 1-m-t_{4}, t_{4}\right) \\
=\int_{0}^{\infty}\left[\left(t_{4}+i \sqrt{x}\right)_{m}\left(t_{4}-i \sqrt{x}\right)_{m}\right]^{2} w\left(x ; t_{1}, t_{2}, 1-m-t_{4}, t_{4}\right) f(x) d x \\
=\int_{0}^{\infty}\left(t_{4}+i \sqrt{x}\right)_{m}\left(t_{4}-i \sqrt{x}\right)_{m} w\left(x ; t_{1}, t_{2}, 1-t_{4}, t_{4}\right) f(x) d x \\
=\int_{0}^{\infty} w\left(x ; t_{1}, t_{2}, 1-t_{4}, t_{4}+m\right) f(x) d x .
\end{gathered}
$$

The uniqueness of the orthonormal polynomials shows that

$$
\frac{W_{n}\left(x, t_{1}, t_{2}, 1-m-t_{4}, t_{4}\right)}{\left(t_{4}+i \sqrt{x}\right)_{m}\left(t_{4}-i \sqrt{x}\right)_{m} W_{n-m}\left(x ; t_{1}, t_{2}, 1-t_{4}, t_{4}+m\right)}
$$

must be a constant, and we have proved (3.10) up to a constant, which can be found by equating the leading terms. The conditions $0<t_{1}, 0<t_{2}$ and $0<t_{4}<1$ can now be removed because (3.10) is a rational function identity in the $t$ parameters. Similarly we treat the cases of the Askey-Wilson polynomials in $\S 4$ and establish (4.4).

\section{ACKNOWLEDGMENTS}

The research of the second author was supported by a research grant from King Saud University, Riyadh, Saudi Arabia. This paper was written while the second author was visiting the Isaac Newton Institute as part of the discrete integrable systems program. He wishes to thank the institute's staff and the program organizers for their hospitality and for the excellent scientific environment. The authors also thank two referees for their comments on an earlier version of this paper.

\section{REFERENCES}

[1] V. E. Adler and A. B. Shabat, On a class of Toda chains, Theor. Math. Phy. 111 (1997), 647-657. MR1472211 (98i:58110)

[2] G. E. Andrews, R. A. Askey, and R. Roy, Special Functions, Cambridge University Press, Cambridge, 1999. MR 1688958 (2000g:33001)

[3] M. Bruschi, F. Calogero, R. Droghei, Proof of certain Diophantine conjectures and identification of a remarkable class of orthogonal polynomials, J. Phys. A: Math. Theor. 40 (2007), 3815-3829. MR 2325078 (2008m:33031)

[4] M. Bruschi, F. Calogero, R. Droghei, Tridiagonal matrices, orthogonal polynomials and conjectures and Diophantine relations. I, J. Phys. A: Math. Theor. 40 (2007), 9793-9817. MR2370544 (2008k:15026)

[5] M. Bruschi, F. Calogero, R. Droghei, Tridiagonal matrices, orthogonal polynomials and conjectures and Diophantine relations. II, J. Phys. A: Math. Theor. 40 (2007), 14759-14772. MR2441873

[6] M. Bruschi, F. Calogero, R. Droghei, Additional recursion relations, factorizations and Diophantine properties associated with the polynomials of the Askey-Scheme, Adv. Math. Phys. 2009, Art. ID 268134, 43 pp. MR2500948

[7] F. Calogero, Isochronous Systems, Oxford University Press, 2008, Appendix C. MR2383111

[8] J. Fields and J. Wimp, Expansions of hypergeometric functions in hypergeometric functions, Math. Comp. 15 (1961), 390-395. MR0125992 (23:A3289)

[9] G. Gasper and M. Rahman, Basic Hypergeometric Series, second edition, Encyclopedia of Mathematics and Its Applications, volume 96, Cambridge University Press, Cambridge, 2004. MR2128719 (2006d:33028) 
[10] M. E. H. Ismail, Classical and Quantum Orthogonal Polynommials in One Variable, Cambridge University Press, Cambridge, 2005. MR2191786 (2007f:33001)

[11] M. E. H. Ismail and D. Stanton, $q$-Taylor theorems, polynomial expansions, and interpolation of entire functions, J. Approximation Theory 123 (2003), 125-146. MR:1985020|(2004g:30040)

[12] R. Koekoek and R. Swarttouw, The Askey-scheme of hypergeometric orthogonal polynomials and its $q$-analogues, Reports of the Faculty of Technical Mathematics and Informatics, no. 9817, Delft University of Technology, Delft, 1998.

[13] A. B. Shabat and R. I. Yamilov, To a transformation theory of two-dimensional integrable systems, Phys. Lett. A 227 (1997) 15-21. MR1435916 (98b:58086)

[14] L. J. Slater, Generalized Hypergeometric Series, Cambridge University Press, Cambridge, 1964.

[15] G. Szegö, Orthogonal Polynomials, 4th edition, Amer. Math. Soc., Providence, RI, 1975. MR0372517 (51:8724)

[16] R. I. Yamilov, Classification of Toda-type scalar lattices, in: Non-linear Evolution Equations and Dynamical Systems, World Scientific, Singapore, 1993.

Department of Mathematics, Imperial College, 180 Queen's Gate, London SW7 2BZ, United Kingdom

E-mail address: ychen@ic.ac.uk

Department of Mathematics, University of Central Florida, Orlando, Florida 32816

E-mail address: ismail@math.ucf.edu 